\title{
Spontaneous Resolution of Primary Small Bowel Volvulus With Oral Contrast
}

\author{
Jasneet Singh Bhullar ${ }^{\mathrm{a}, \mathrm{b}}$, Peter Papapetrou ${ }^{\mathrm{a}}$, Gokulakkrishna Subhas ${ }^{\mathrm{a}}$, \\ Natasha Gupta ${ }^{a}$, Susan Semana, Vijay Kumar Mittal ${ }^{\mathrm{a}}$
}

\begin{abstract}
We report a case of 49 year-old male who presented to the emergency room with abdominal pain. $\mathrm{CT}$ of the abdomen revealed a small bowel volvulus. After ingestion of the contrast dye used for the CT imaging study, he experienced resolution of his presenting symptoms. Furthermore, his follow up abdominal radiograph done 1 hour later showed a decrease in the number of distended small bowel loops, corresponding to an immediate improvement in his condition. This is the first report of spontaneous resolution of primary small bowel volvulus after ingestion of oral contrast. This case can be used for further investigation of a therapeutic role of oral contrast in the management of primary small bowel volvulus.
\end{abstract}

Keywords: Small bowel volvulus; Spontaneous resolution; Oral contrast

\section{Introduction}

Volvulus is a rare cause of small bowel obstruction, with the majority of volvulus cases occurring in the sigmoid colon. However, small bowel volvulus can be worrisome if not diagnosed and treated early. Mortality rates are high, between 9-35\%, and even higher if necrosis is present in late presentation. Preferred management is computed tomography (CT) scan of the abdomen for diagnosis, followed by laparotomy and bowel resection if non viable bowel is identified.

\section{Case Report}

A 49 year-old male was presented to the emergency room

Manuscript accepted for publication June 6, 2012

${ }^{a}$ Department of Surgery,Providence Hospital and Medical Centers, 16001

West Nine Mile Road, Southfield, MI, USA

${ }^{\mathrm{b}}$ Corresponding author: Jasneet Singh Bhullar.

Email: drjsbhullar@gmail.com

doi:10.4021/jes67w with a five hour history of severe abdominal pain. His pain had a sudden onset in the mid-abdomen, and was stabbing in nature. The pain was also accompanied by abdominal cramping, nausea and diaphoresis. His only previous surgery was a left inguinal hernia repair. Physical examination revealed the patient to be diaphoretic with a mildly distended generalized tender abdomen and positive bowel sounds. Laboratory investigations showed an elevated WBC count of $16,300 / \mu \mathrm{L}$ with a left shift of $82.2 \%$ and lactic acid of 2.3 $\mathrm{mmol} / \mathrm{L}$. An antero-posterior plain abdominal $\mathrm{X}$ ray showed a picture of small bowel obstruction with multiple distended small bowel loops and air-fluid levels (Fig. 1). Subsequently, a contrast enhanced CT of the abdomen and pelvis was done, which showed a partial small bowel obstruction caused by small bowel volvulus (SBV) in the mid abdomen with characteristic 'whirl' sign (Fig. 2A, B). The patient reported that upon drinking the oral contrast, $100 \mathrm{~mL}$ of Isovue-370, the abdominal pain resolved within 1 hour, and was followed by having a bowel movement. The patient was able to tell the exact time when he felt the movements of bowel in the abdomen and complete resolution of the abdominal pain. Reexamination two hours later revealed a non tender and non distended abdomen. Follow up lactic acid level decreased to 1.1 $\mathrm{mmol} / \mathrm{L}$, and $\mathrm{WBC}$ count decreased to $11,200 / \mu \mathrm{L}$. A repeat antero-posterior plain abdominal $\mathrm{X}$-ray revealed a decrease

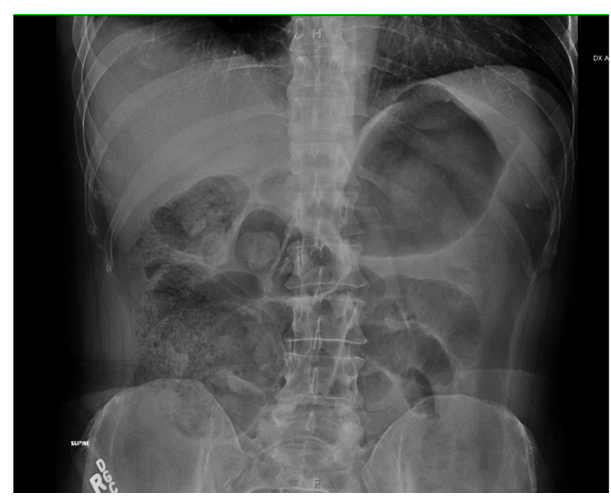

Figure 1. Antero-posterior plain abdominal $\mathrm{X}$ ray at presentation, showing distension of small bowel loops with air fluid levels. 


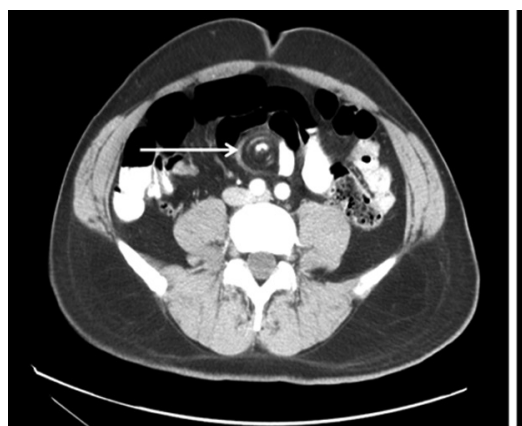

A

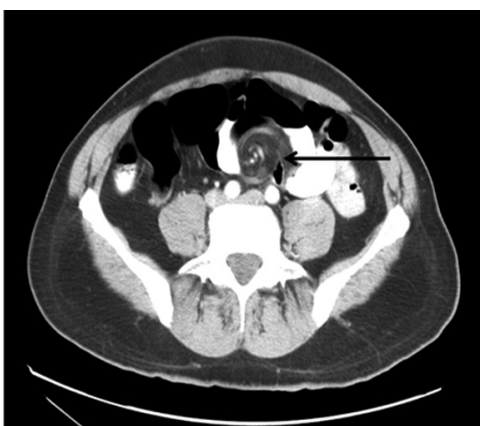

B

Figure 2. A and B: Contrast enhanced CT axial view (oral and intravenous contrast), showing dilated small bowel loops with swirling appearance of mesenteric vessels "Whirl sign" (marked with arrows), and small bowel in the mid abdomen consistent with small bowel volvulus.

in the number of distended small bowel loops, as well airfluid levels (Fig. 3). The patient was discharged the same day and subsequent follow up revealed no residual symptoms.

\section{Discussion}

Volvulus is the Latin word for rolled up or twisted and is derived from the verb 'volvere', meaning to roll or turn about. By definition, volvulus is an abnormal twisting of the intestine which can impair the blood flow to the intestine. Volvulus can lead to gangrene and death of that segment of the gastrointestinal tract, intestinal obstruction, perforation of the intestine, and peritonitis.

Small-bowel volvulus is the twisting of small bowel about its own mesentery, which leads to a small bowel obstruction [1].This causes a narrowing of the bowel lumen, as well as restricting blood supply to the bowel. Patients with SBV present with an acute abdomen, though it is relatively rare in adults compared to volvulus of the colon and cecum [2]. Volvulus is the small bowel, accounts for $3-6 \%$ of all

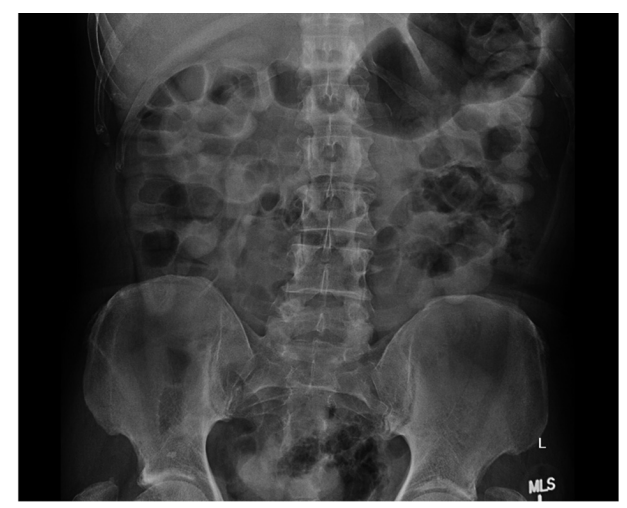

Figure 3. Antero-posterior plain abdominal $X$ ray 2 hours after pain resolution, showing contrast in the bowel with decrease in the number of distended small bowel loops. cases of small bowel obstruction in Western nations, with an incidence of $1.7-5.7$ per 100,000 individuals [3]. In the adult population, SBV is classified as primary and secondary. Primary SBV refers to cases where there is a normal anatomic cavity with no predisposing factors. Secondary SBV refers to cases where there is a congenital or acquired lesion in the abdominal cavity, such as midgut nonrotations or surgical adhesions [2]. Primary SBV is more common in Asia and Africa, where there are prolonged periods of fasting followed by ingestion of large quantities of fiber [3]. Secondary SBV is more common in western countries, and is caused by adhesions secondary to abdominal surgery. As a result of SBV, the patient could develop bowel ischemia, causing necrosis of the small bowel, which is a surgical emergency. SBV causes mortality in $9-35 \%$ of cases, and when there is necrosis of the small bowel the mortality is $20-100 \%$ [2, 4]. Conventional management involves urgent laparotomy to resect portions of nonviable small bowel [5].

The most common site of volvulus is the sigmoid colon, accounting for $2-5 \%$ of all colonic obstructions [6]. A rare site of volvulus is the stomach, which can result from a loosely adherent stomach rotating upon itself in the peritoneal cavity [7].

SBV presents as abdominal pain, distension, nausea and vomiting; SBV may also be associated with fever and an elevated WBC count $(>18,000)$ when necrotic bowel loops are present [8]. Liao et al. claim that a predisposing factor that can lead to small bowel volvulus is a bowel with a long mobile mesentery and a short mesenteric root [8]. The investigation of choice for diagnosis of SBV is CT scan, which can show a "whirl" sign of twisted mesentery (Fig. 3A, B) and "peacock's tail" sign of a torsed bowel [3]. CT scan is also the preferred imaging study as it is accurate in identifying ischemic bowel, compared to abdominal radiographs [3].

Once SBV is diagnosed on CT scan, the patient undergoes surgery to determine if there is bowel necrosis and fix the volvulus. Intra-operatively if bowel necrosis is discovered, the necrotic bowel is resected and the viable ends of 
the bowel are anastomosed. One critical factor in patient outcome is the time from emergency department evaluation to surgery. Ruiz-Tovar et al. discovered that there were more necrotic bowel loops if the patient underwent laparotomy more than 24 hours after emergency department evaluation [3]. Therefore a high degree of suspicion with early diagnosis is critical.

Our patient presented with clinical signs and symptoms of SBV without necrosis. This is supported by the fact he had a sudden onset of sharp, mid-abdominal pain that led to higher suspicion of volvulus. His early presentation to the emergency room and the fact that he was afebrile with a WBC count of 16,300 indicated that he probably did not have bowel necrosis. Our case is unique in that upon drinking the oral contrast for diagnostic CT scan, the patient's symptoms were relieved clinically and radiographically. This could indicate a therapeutic benefit of oral contrast in relieving SBV, which could make surgical intervention unnecessary. Although the oral contrast's effect has not been proven, it is hypothesized that the contrast was able to devolvulate the small bowel by mechanical means and being osmotic in nature it stimulated peristalsis. As the contrast passed through the small bowel, the bowel reassumed its untorsed configuration.

Our reported case shows evidence in which the patient's primary SBV was relieved immediately after drinking oral contrast for CT scan. His symptoms, clinical findings as well as follow up investigations revealed a high degree of resolution. Future studies looking into the therapeutic role of oral contrast in managing SBV would be needed to further investigate its efficacy.

\section{Conclusion}

Small bowel volvulus (SBV) is a very rare cause of small bowel obstruction in adults requiring prompt surgical treatment. Our reported case shows evidence in which the patient's primary SBV was relieved after drinking oral contrast for CT scan.

\section{Disclosures}

All authors have nothing to disclose.

\section{References}

1. Katis PG, Dias SM. Volvulus: a rare twist on small-bowel obstruction. CMAJ. 2004;171(7):728.

2. Roggo A, Ottinger LW. Acute small bowel volvulus in adults. A sporadic form of strangulating intestinal obstruction. Ann Surg. 1992;216(2):135-141.

3. Ruiz-Tovar J, Morales V, Sanjuanbenito A, Lobo E, Martinez-Molina E. Volvulus of the small bowel in adults. Am Surg. 2009;75(12):1179-1182.

4. Iwuagwu O, Deans GT. Small bowel volvulus: a review. J R Coll Surg Edinb. 1999;44(3):150-155.

5. Talbot $\mathrm{CH}$. Volvulus of the small intestine in adults. Gut. 1960;1:76-80.

6. Atamanalp SS. Sigmoid Volvulus. EAJM 2010; 42: 142-7.

7. Hsu YC, Perng CL, Chen CK, Tsai JJ, Lin HJ. Conservative management of chronic gastric volvulus: 44 cases over 5 years. World J Gastroenterol. 2010;16(33):42004205.

8. Liao YC, Chiu HH, Huang TC. Education and imaging. Gastrointestinal: small bowel volvulus. J Gastroenterol Hepatol. 2008;23(10):1625. 